\title{
1 Variation of the vaginal microbiome during and after pregnancy in \\ 2 Chinese women
}

3 Xiaoai Zhang ${ }^{1, \#, a}$, Qingzhi Zhai ${ }^{2, \#, b}$, Jinfeng Wang ${ }^{3, \#, c}$, Xiuling Ma ${ }^{3, \mathrm{~d}}$, Bo Xing ${ }^{1, \mathrm{e}}$,

4 Hang Fan ${ }^{1, \mathrm{f}}$, Zhiying Gao ${ }^{2, \mathrm{~g}}$, Fangqing Zhao ${ }^{3,5,6,7 * \text {, h }}$, Wei Liu ${ }^{1,4, *, \mathrm{i}}$

$5 \quad{ }^{1}$ State Key Laboratory of Pathogen and Biosecurity, Beijing Institute of Microbiology

6 and Epidemiology, Beijing 100071, China

$7{ }^{2}$ Department of Obstetrics and Gynecology, First Medical Center, The General

8 Hospital of the People's Liberation Army, Beijing 100853, China

$9 \quad{ }^{3}$ Computational Genomics Lab, Beijing Institutes of Life Science, Chinese Academy

10 of Sciences, Beijing 100101, China

$11{ }^{4}$ Beijing Key Laboratory of Vector Borne and Natural Focus Infectious Disease,

12 Beijing 100071, China

$13{ }^{5}$ Center for Excellence in Animal Evolution and Genetics, Chinese Academy of

14 Sciences, Kunming 650223, China

$15{ }^{6}$ Key Laboratory of Systems Biology, Hangzhou Institute for Advanced Study,

16 University of Chinese Academy of Sciences, Chinese Academy of Sciences, Hangzhou,

17 China

$18{ }^{7}$ University of Chinese Academy of Sciences, Beijing 100049, China

$20 \quad$ \# Equal contribution.

$21 *$ Corresponding authors.

22 E-mail: zhfq@biols.ac.cn (Zhao F), liuwei@bmi.ac.cn (Liu W). 
${ }^{a}$ ORCID: 0000-0002-9505-1307

24 b ORCID: 0000-0002-7328-2948

25 ' ORCID: 0000-0003-4909-2738

26 d ORCID: 0000-0003-3225-0672

27 e ORCID: 0000-0001-8906-362X

28 f ORCID: 0000-0002-7927-1684

29 g ORCID: 0000-0002-5543-8961

$30{ }^{\mathrm{h}}$ ORCID: 0000-0002-6216-1235

31 i ORCID: 0000-0002-9302-8170

32 Running title: Zhang X et al / The Vaginal Microbiota of Chinese Pregnant Women

33 Word count: 5724

34 Figures: 5

35 Tables: 0

36 Supplementary figures: 8

37 Supplementary tables: 3 


\section{Abstract}

46 A more complete profiling of vaginal microbial communities and their variability enables a more accurate description of women microbiome. However, there is a distinct lack of information regarding the Chinese women. Composition of the vaginal microbiota during pregnancy and 6 weeks postpartum of 454 Chinese women thus was characterized in this study by sequencing V3-V4 regions of the $16 \mathrm{~S}$ ribosomal RNA (rRNA). It showed that the vaginal microbiome varied during pregnancy and postpartum in response to abortion history, hypertensive disorders (HBP), delivery mode and maternal age. Co-variation of 21 bacterial taxa, including Lactobacillus and two of its species, may account for the common characteristics of vaginal microbiome under different medical histories and pregnancy outcomes. On the contrary, discriminant bacteria were significantly different between premature rupture of membranes related preterm birth (PROM-PTB) and non-PROM related PTB, and community state type (CST) I without any predominant Lactobacillus species in microbiota was more prevalent during pregnancy in PROM-PTB, suggesting that specific bacteria could be considered to distinguish different types of PTB. Through adding the data from Chinese women, the study will enrich the knowledge of human microbiome and likewise contribute to a better understanding of the association between the vaginal microbiome and reproductive health.

KEYWORDS: Chinese pregnant women; Vaginal microbiota; Postpartum; 


\section{Introduction}

The Human Microbiome Project (HMP) has released the human microbiome data derived from thousands of individuals [1]. As an important part of the HMP and one of the most prosperous community in human body, vaginal microbiome has received much attention [2,3]. Over recent years, evidence is accumulating that vaginal microbiome is key to women's health and a healthy pregnancy, and people gradually realize that the health status largely depends on this microbial community harboring more beneficial commensals or pathogens [4-9]. Most of the studies have introduced the compositions and shifts of the vaginal microbiome in women of African, European and American population [10, 11], however, there is a lack of knowledge about the vaginal microbiome in Chinese pregnant women.

In China, nearly half of the births were delivered by caesarean section in 2007-2008, and the rate was even close to $60 \%$ in some cities [12-14]. With start of two-child policy in China from 2015, healthcare providers are facing the greater challenges of either a higher cesarean section rate, or an advanced maternal age, or both. In addition, in China, more than 1 million preterm infants are born per year, second only to India [15-17]. All these serious situations in obstetrics and gynecology have become major public health concerns in China and around the world. Previous studies have shown that the composition of vaginal microbiome was associated with pregnancy, delivery mode, maternal age, and preterm [4-8]. A more complete characterization of vaginal microbiome and its variability with pregnancy, delivery mode, or specific characters of pregnant women will enable a more accurate diagnosis 
89

90

91

of women who truly possess an abnormal vaginal microbiome.

In recent years, our understanding of vaginal microbiome has broadened by using cultivation intendent high-throughput sequencing. Ravel et al. performed a 16S ribosomal RNA (rRNA) gene survey on vaginal samples from 396 North American women represented four ethnic groups [18]. They divided the vaginal microbiome into five community state types (CSTs) based on the species of dominant bacteria. CST I, CST II, CST III and CST V was found to be predominated by Lactobacillus crispatus, Lactobacillus gasseri, Lactobacillus iners, and Lactobacillus jensenii, respectively, and CST IV was defined as lacking Lactobacillus spp. and comprising a diverse set of strict and facultative anaerobes. Following longitudinal study in white, black, and Hispanic reproductive-age women over 16 weeks, it was suggested that CSTs was dynamic in some women, but relatively stable in the others [19]. Thereafter, CSTs were widely used in the studies of the association between the vaginal microbiome and reproductive health for its effective handle.

To date, some studies linking the vaginal microbiome to preterm birth (PTB) have yielded mixed and even discordant results [4-7]. Romero et al. found no association between vaginal microbiome and either premature rupture of membranes (PROM) related or non-PROM related PTB in a predominantly African American cohort [4]. One study reported that CST V were associated with clinically heterogeneous PTB in two predominantly Caucasian populations [5]. Lindsay et al detected a significant positive association between CST III and non-PROM related PTB in high-risk Caucasian, Asian, and Black pregnant women [6]. Recently, 
medRxiv preprint doi: https://doi.org/10.1101/2020.07.07.20148536; this version posted July 10, 2020. The copyright holder for this preprint (which was not certified by peer review) is the author/funder, who has granted medRxiv a license to display the preprint in perpetuity.

All rights reserved. No reuse allowed without permission.

111 Digiulio et al. replicated their previously reported associations [5] between less

112 Lactobacillus or more Garderella and clinically heterogeneous PTB in the low risk

113 Stanford cohort [7]. However, their previously hypothesized association [5] between

114 PTB and more Ureaplasma was not replicated in their following study [7]. Resolving

115 these mixed and even discordant findings in prior studies requires the need for

116 investigation of the microbiome with different types of PTB and PROM

117 simultaneously in a more comprehensive design and a larger sample size of cohort.

118 In this study, by using sequencing of $16 \mathrm{~S}$ rRNA gene amplicons, we

119 characterized and compared the vaginal microbiome community of Chinese pregnant

120 women according to multiple factors such as delivery mode, maternal age, abortion

121 history, pre-pregnancy maternal weight status, and pregnancy complications during

122 pregnancy and postpartum. Furthermore, the relationships between vaginal

123 microbiome and the clinical features of adverse pregnancy outcomes, in particular

124 PTB, were analyzed. We wanted to expand the current understanding for the vaginal

125 microbiome in Chinese pregnant women and whether the microbial community shifts

126 over time or under certain conditions.

\section{Results}

128 From July to December 2016, totally 474 pregnant Chinese women attending the

129 Department of Obstetrics at the 301 Hospital (Beijing) for regular check-ups were

130 enrolled in this study. Of the 474 volunteers recruited, 20 pregnant women were

131 excluded because of medical complications (from fetal or pregnant women) requiring

132 induction of labor. Of 454 women included in final analyses, vaginal swabs of 142 
133

pregnant women were sampled at early stage of pregnancy ( $\leq 18$ gestational weeks), 207 were sampled at late stage of pregnancy $(27 \leq$ gestational weeks $<42)$, and 98 were sampled 6 weeks postpartum (Supplementary Table S1). Using 16S rRNA-based sequencing we obtained a total of 27,171,551 high-quality sequences from the vaginal samples of Chinese pregnant women, with median 59,912 and interquartile range 57,311-62,351 sequences per sample. Totally 6343 operational taxonomic units (OTUs) were generated with $\geq 97 \%$ sequence similarity.

\section{The vaginal microbial community shifts significantly from pregnancy to} postpartum

Alpha diversity, as quantified by the Chao1 index, was significantly higher $(P<0.001)$ in the vaginal microbiome postpartum than pregnancy (Figure 1A). The other four diversity indices showed consistent results (Supplementary Figure S1A-D), indicating that the microbial richness may have increased dramatically after delivery. Similarly, significant differences were also found in $\beta$ diversities based on the weighted UniFrac dissimilarities (ANOSIM R $=0.511, P=0.001$ ) between pregnancy and postpartum (Figure 1B and Supplementary Figure S2A-D). Moreover, vaginal samples from pregnancy clustered more closely.

For each taxonomic category, the difference was rather large. At the phylum level, only the relative abundance of Firmicutes was higher during pregnancy than postpartum, while more Actinobacteria, Proteobacteria, Bacteroidetes and other 7 phyla appeared in postpartum women (Figure 1C). Lactobacillus genus and 5 of its species L. crispatus, L. gasseri, L. iner, L. jensenii, and L. reuteri were significantly 
medRxiv preprint doi: https://doi.org/10.1101/2020.07.07.20148536; this version posted July 10, 2020. The copyright holder for this preprint (which was not certified by peer review) is the author/funder, who has granted medRxiv a license to display the preprint in perpetuity.

All rights reserved. No reuse allowed without permission.

155 higher, and dozens of genera such as Prevotella, Atopobium, Acinetobacter and

156 Sneathia were significantly lower during pregnancy (Figure 1D and Supplementary

157 Table S2).

158 In view of the obvious difference of Lactobacillus before and after birth, we

159 identified four CSTs from the vaginal microbiome of Chinese pregnant women

160 (Supplementary Figure S3 and Supplementary Table S3). CST I and CST III were $L$.

161 crispatus and L. iners dominated, respectively. Both CST IV-A and CST IV-B were

162 non-Lactobacillus dominated, and the difference between them is that some women in

163 the former still retained a certain amount of L. crispatus, L. iners, L. jensenii and/or $L$.

164 gasseri, while the latter contained more Gardnerella. The most prevalent CSTs

165 observed in Chinese pregnant women was CST I (41.9\%), followed by CST IV-A

166 (31.1\%), CST III (18.7\%), and CST IV-B (8.3\%). Samples of pregnancy source

167 distributed in all types of CSTs, but mainly in CST I and CST III. In contrast,

168 postpartum samples were basically found in CST IV, especially had greater numbers

169 of CST IV-A $\left(\chi^{2}=92.08, P<0.001\right)$. These results indicate that in Chinese women,

170 vaginal microbiome lacks a portion of Lactobacillus after delivery, which makes the

171 microbial diversity becoming higher, but it is not completely occupied by harmful

172 bacteria such as Gardnerella.

173 Neither significant difference in the $\alpha$ and $\beta$ diversity (weighted UniFrac,

174 ANOSIM R $=-0.009, P=0.693$ ) (Supplementary Figure S4A-F), nor bacterial taxa

175 differed in relative abundance was observed between early and late stages of

176 pregnancy $(P>0.05$ after FDR correction, Supplementary Figure S4G-H). 

abortion history

During pregnancy, the $\alpha$-diversity of vaginal microbiome was significantly higher in pregnant women with hypertensive disorders (HBP) than without $(P=0.037$, Figure between HBP and control groups based on linear discriminant analysis effect size (LEfSe) analysis (Figure 2B), and almost all of these discriminating taxa were enriched rather than depleted in the case group. Suffering from HBP was not associated with a reduction in the relative abundance of Lactobacillus, but was accompanied by an increase in proportion of genera such as Gardnerella, Atopobium, and Sneathia. The results reveal that HBP may have some impact on the vaginal microbiota during pregnancy, causing abundance changes in many bacteria, and part of which was consistent with that happened after delivery (Figure 1D).

UniFrac (ANOSIM R $=0.054, P=0.015$ ) between pregnant women with and without history of abortion (Figure 2C). Samples collected from women who did not have abortion history were more intensive, while those with a history of abortion were 
199

200

201

202

203

204

205

206

207

microbiota would be similar, on the contrary, abortion may increase the heterogeneities between individuals, resulting in completely different community structure. Because there were more discriminating taxa that have undergone significant changes, the impress of HBP on vaginal microbiome seems to be greater than the history of abortion. This may be partly due to the fact that HBP occurring during pregnancy has a greater impact on maternal physiology which would immediately transfer to the microbiota, while the previous abortion leaves only a slight trace.

Although there was no significant difference in $\alpha$ and $\beta$ diversity, several characteristic bacteria corresponding to four factors including delivery mode, maternal age, gestational diabetes mellitus, and hypothyroidism could be found in the vaginal microbiome during pregnancy (Supplementary Figure S5).

\section{The vaginal microbiome postpartum varied by delivery mode and maternal age}

Postpartum vaginal microbiome showed a strong association with delivery mode.

After delivery, the microbial diversity of vaginal microbiome in the woman who delivered by cesarean section was significantly higher than vaginally delivered women $(P<0.001$, Figure 3A). Significant difference was also found in community structure based on the weighted UniFrac between women who delivered cesarean section and vaginally, with the latter had a smaller $\beta$ diversity (ANOSIM R $=0.080, P$ $=0.034$, Figure 3B). It seems that the vaginal microbiota of the pregnant women who delivered vaginally is dominated by fewer microbial species after delivery, which also has a more similar community structure to each other. The discriminating taxa 
221 identified from the LEfSe analysis further reflect that the Lactobacillus, which are

222 resident of healthy women's vagina, were more abundant in the postpartum vagina of

223 the pregnant women who delivered vaginally (Figure 3C).

We found maternal age is another factor associated with the variation of postpartum vaginal microbiome. Samples collected from women with advanced and young age grouped into two distinct clusters (ANOSIM R $=0.149, P=0.022$, Figure 3C), and the elder formed a more consistent community structure. Based on LEfSe analysis, the vaginal microbiome of the women with advanced age were lacking Lactobacillus genus, and its two species $L$. iners and L. reuteri after delivery (Figure 3E). Besides delivery mode and maternal age, dramatic shifts in relative abundance of some bacteria were observed in the grouping of three other factors including abortion history, maternal pre-pregnancy weight status and pregnancy complications (Supplementary Figure S6).

Identification of common key taxa accounting for the variation of vaginal

microbiome

Totally, the relative abundances of 163 bacterial taxa from phylum to genus level varied significantly between groups during pregnancy and postpartum based on LEfSe analysis (Supplementary Figure S7). These bacterial taxa rarely had overlaps across different factors, and most of the discriminating taxa were unique to each abnormal factor. Moreover, the discriminating taxa of the same factor had no consistency between prenatal and postpartum. Certain bacterial taxa associated with 
medRxiv preprint doi: https://doi.org/10.1101/2020.07.07.20148536; this version posted July 10, 2020. The copyright holder for this preprint

(which was not certified by peer review) is the author/funder, who has granted medRxiv a license to display the preprint in perpetuity.

All rights reserved. No reuse allowed without permission.

243 distinction of the discriminating taxa and the abundance divergence of the same taxa

244 reflected the large differences of vaginal microbial communities during pregnancy

245 and postpartum as well as their response to various abnormal factors.

246 Even though, several common taxa accounting for the variation of vaginal

247 microbiome can still be identified from the 163 discriminating taxa. Relative

248 abundance of 21 bacterial taxa was simultaneously altered in at least 2 of the 10

249 comparisons (Figure 4A). Thirteen bacterial taxa shifted in the same direction in two

250 comparisons, and significant differences were consistently recorded for 8 genera

251 Propionibacterium, Rheinheimera, Butyricimonas, Lactobacillus, Sneathia, Bulleidia,

252 Cellulosilyticum, and Nosocomiicoccus. Common taxa were more likely to present

253 with the two factors of delivery mode and maternal age, especially the postpartum

254 microbiome associated with maternal age. In addition, Lactobacillaceae family

255 decreased in abundance in pregnant women who delivered cesarean section and

256 advanced maternal age (Figure 4B). The relative abundance of $L$. iners was lowered

257 in pregnant women who delivered without abortion history during pregnancy and

258 those with advanced maternal age in postpartum (Figure 4C). The abundances of $L$.

259 reuteri depleted in women with abortion history and advanced maternal age in

260 postpartum (Figure 4D). These results suggest that vaginal delivery, young maternal

261 age, and without abortion history perhaps are more appropriate for the growth of

262 Lactobacillus in maternal vagina, which should be good for women's health. Some

263 vaginal microbes showed the same response to different abnormal factors and may be

264 more susceptible to the influence, so these bacteria together with the maternal age, 
265 require special care.

266 Variations of CSTs associated with multiple factors during pregnancy and

\section{7 postpartum}

268 To facilitate the comparison of the relationships between vaginal microbiome and

269 different factors in different periods, we once again examined the distribution of CSTs

270 (Figure 5 and Supplementary Table S3). We found the largest discrimination before

271 and after delivery was that CST I and CST IV-A had the highest proportions,

272 respectively. In the vaginal microbiome during pregnancy, the ratios of CST IV-A in

273 the groups of cesarean section, advanced maternal age, with abortion history,

274 overweight, with hypertensive disorders, and with hypothyroidism were remarkably

275 higher than those of corresponding groups. It is worth noting the difference of CSTs

276 composition between pregnant women who have normal (term) delivery compared to

277 PTB and PROM. The term delivery group during pregnancy showed a lower CST

278 IV-A/CST I ratio (0.35), and such ratio increased to 0.53 if PROM occurred even

279 though there was no premature birth at last (term-PROM). In addition, a high CST

280 IV-A/CST I ratio (1.00) was shown in the group of pregnant women who have

281 delivered PTB, and such ratio (2.00) was particularly high in PROM complicated with

282 PTB (PROM-preterm) during pregnancy. In the vaginal microbiome after delivery,

283 the situation of more CST IV-A was further exacerbated, with the only exception

284 being hypertensive disorders. The divergence between term and PTB had not

285 disappeared, and CST I was not even detected in both PROM-preterm and preterm.

286 To further investigate the connections between vaginal microbiome and PTB and 
medRxiv preprint doi: https://doi.org/10.1101/2020.07.07.20148536; this version posted July 10, 2020. The copyright holder for this preprint (which was not certified by peer review) is the author/funder, who has granted medRxiv a license to display the preprint in perpetuity.

All rights reserved. No reuse allowed without permission.

287

288

289

290

291

292

293

294

295

296

297

298

299

300

301

302

303

304

305

306

307

308

PROM, microbial composition was compared between each group during pregnancy and postpartum. The relative abundances of 68 bacterial taxa, including 1 phylum, 3 classes, 9 orders, 18 families, 34 genera, and 3 species varied significantly between groups (Supplementary Figure S8A-G). During pregnancy, 6 bacterial taxa, including Lactobacillus buchneri and Lactobacillus coryniformis, and 2 bacterial taxa were increased in abundance in pregnant women with preterm or PROM neonate (Supplementary Figure S8A-B). When we stratified women who delivered preterm by PROM status, 3 bacterial taxa were associated with PROM related preterm (Supplementary Figure S8C), and 16 bacterial taxa were associated with non-PROM related preterm (Supplementary Figure S8D). Among women who delivered preterm, the abundances of 4 bacterial taxa were significantly higher in those with PROM compared to those without PROM (Supplementary Figure S8E). These discriminant bacteria in the vaginal microbiome, with significant changes in prenatal abundance, may be served as candidate biomarkers for predicting preterm birth. In postpartum, 20 bacterial taxa, such as L. gasseri, were more abundant in preterm group, while 9 bacterial taxa, such as Bacillus, were more abundant in term group (Supplementary Figure S8F). Significant differences were also recorded for the 19 bacterial taxa between pregnant women with and without PROM (Supplementary Figure S8G).

\section{Discussion}

Both cross-sectional and longitudinal studies reported that the vaginal microbiome during pregnancy was less diverse and more stable than that of postpartum [20]. Chinese women have not been well represented before. To this end, this study 
medRxiv preprint doi: https://doi.org/10.1101/2020.07.07.20148536; this version posted July 10, 2020. The copyright holder for this preprint

(which was not certified by peer review) is the author/funder, who has granted medRxiv a license to display the preprint in perpetuity.

All rights reserved. No reuse allowed without permission.

309

310

311

312

313

314

315

316

317

characterized the vaginal microbiome of the largest cohort of Chinese women to date.

Consistent with previous studies in women of African, Hispanic or European [10, 20], we also found that diversity and composition of vaginal microbial communities are relatively stable at early and late time points in pregnancy and dramatically changed in postpartum period to be less Lactobacillus dominant in Chinese population. Furthermore, we found that the vaginal microbiome changed in response to abortion history and hypertensive disorders during pregnancy and delivery mode and maternal age in the postpartum period.

Identification of key taxa within the diverse vaginal microbiome is of great importance because they may pose differing risks for adverse health outcomes in pregnant women. Our study identified a series of significant bacterial taxa that differed significantly in relative abundance according to the delivery mode, maternal age, history of abortion, and pregnancy complications. Notably, we also found several key taxa that differed significantly in relative abundance in at least two comparisons. Considering that these key taxa were constantly mentioned to be associated with women's health in previous studies [6,11], which point toward them as taxa of particular importance for study in the future. Other taxa, such as Propionibacterium enriched in cesarean section newborns [21], Butyricimonas enriched in the patients of Autism spectrum disorder [22] and depleted in patients with histamine intolerance [23] and thyroid cancer [24], and Bulleidia appears more frequently in patients with esophageal squamous cell carcinoma [25], also merit additional investigation.

CST is widely used in the studies of vaginal microbiome to deal with 
331

332

inter-subject and/or intra-subject variability of women [26]. Through a comparison of this new dataset with existing datasets from Caucasian women and other countries, it revealed that the prevalence of CSTs may be different across populations. Using a large sample size of 396 North American women from four ethnic groups, including white, black, Hispanic, and Asian, Ravel et al. established five CSTs [18]. Huang et al reported the identification of four CSTs from 34 Chinese women during different pregnancy stages [27]. Considering that there is a distinct lack of information regarding the CSTs in Chinese pregnant women, our results from a population of large Chinese women contribute to a more comprehensive understanding of CSTs of Chinese pregnant women. Similar to Huang et al, we also identified four CSTs previously described: I (41.9\%), III (18.7\%), IV-A (31.1\%), and CST IV-B (8.3\%), but did not find CST II and CST V in our study population. The possible reasons of lack some CSTs in Chinese women and their difference to Caucasian women may be their living environment or ethnicity.

Unexpectedly, we found that changes in the vaginal microbiome community is also related to GDM, which has not been reported in neither Chinese nor Caucasian population. Compared with healthy pregnant women, we found that women suffered from GDM had a higher proportion of CST I during pregnancy, while the proportion of non-Lactobacillus dominated CST IV was lower. After delivery, the proportion of CST I in GDM women was still higher than that of healthy women. But unlike during pregnancy, the proportion of CST IV-B which contains more Gardnerella and is generally considered to be unfavorable to women's vaginal health [18], increased 
353

remarkably in postpartum women who have had GDM. These results suggest that

GDM may be more harmful to postpartum vaginal microbiome and health than to prenatal, and more attentions should be paid to the postpartum health of this female population.

In this study, we present a study design with the challenge of mixed and even discordant findings in some studies linking the vaginal microbiome to PTB in mind. Consistent with the Romero's study [4], we found no differences in the frequency of observed CSTs between women who delivered at term and those who delivered preterm. However, compared to their results which did not find key taxa differed in relative abundance [4], we found bacterial taxa were significantly different between PROM related PTB and non-PROM related PTB, indicating that different types of PTB had specific bacterial taxa. Some of contributing taxa such as Gardnerella, Ureaplasma and/or Megasphaera could be considered for developing predictive models in Chinese population. We can replicate previously reported association between more Gardnerella, Ureaplasma or Megasphaera and clinically heterogeneous PTB in cohorts of predominantly African descent in postpartum stage $[5,7]$. The previously hypothesized associations between less Lactobacillus abundance and PTB in cohorts of predominantly African descent, more L. iners abundance and PTB in cohort of predominantly European descent were not replicated in our study [5-7]. The differences between previous studies and our results strongly suggest that PBT-microbiome associations may be racially-dependent. Further population-specific studies are needed to assess the impacts of the association 
375 between vaginal microbiome and PTB and to identify population-specific key taxa.

376 Although the present study represents the most extensive examination of the

377 vaginal microbiome of Chinese pregnant women to date, in reviewing of our results,

378 several limitations should be considered. First, this is a cross-sectional study in which

379 samples were obtained at a single time point. Looking forward, prospective

380 longitudinal studies are also needed to confirmed our results. Secondly, studies using

381 metagenomic sequencing are needed in the future to provide more detailed

382 information about the function and changes in the vaginal microbiome. In addition,

383 absence of a concurrent analysis of host factors is a likely reason for the variable

384 conclusions [28].

385 Conclusions

386 This is the first study to characterize a Chinese cohort of this size. We identified

387 measurable differences in vaginal microbiome of Chinese pregnant women according

388 to delivery mode, maternal age, and history of abortion and hypertensive disorders,

389 with possible consequences for both short- and long-term health. The study also

390 demonstrated that PBT-microbiome associations are population-dependent and

391 reveals new insights into ethnic and biogeographical effects upon the association

392 between PTB and vaginal microbiome. With the realization that our understanding of

393 the microbiome is as good as the diversity of people sampled, this dataset will provide

394 valuable information for future research and contribute to a more comprehensive

395 understanding of the correlation between bacterial community and Chinese pregnant

396 women. 


\section{Materials and methods}

398

399

400

401

402

403

404

405

406

407

408

409

410

411

412

413

414

415

\section{Patients and samples}

The study was performed with the approval of the Ethical Committee of Beijing Institute of Microbiology and Epidemiology and conducted according to the principles expressed in the Declaration of Helsinki. From July to December 2016, totally 474 pregnant Chinese women attending the Department of Obstetrics at the 301 Hospital (Beijing) for regular check-ups were enrolled in this study. All participants provided informed consent. Information regarding demographic characteristics, medical history, clinical manifestation, laboratory test results were prospectively collected using a standard questionnaire. For each participant, a cotton swab was used to collect discharge from posterior vagina. The samples were stored at $-20^{\circ} \mathrm{C}$ upon collection. All samples were stored at $-80^{\circ} \mathrm{C}$ within 4 hours until metagenomic DNA extraction.

\section{Processing of microbial samples}

Microbial DNA was extracted from vaginal swabs and sterile water using the DNA extraction kit (Cat No. 69504, Qiagen, Hilden, Germany) according to manufacturer's protocols. DNA concentration and purity were measured by Qubit 3.0 (Cat No. Q33216, Thermo Fisher Scientific, Waltham, MA). DNA was amplified using polymerase chain reaction (PCR) with a pair of barcoded primers (341F: CCTAYGGGRBGCASCAG; 806R: GGACTACNNGGGTATCTAAT) targeting the V3-V4 region of $16 \mathrm{~S}$ rRNA gene. Each PCR reaction was conducted in a $30 \mu \mathrm{L}$ reaction system with $15 \mu \mathrm{L}$ of Phusion High-Fidelity PCR Master Mix (Cat No. 
medRxiv preprint doi: https://doi.org/10.1101/2020.07.07.20148536; this version posted July 10, 2020. The copyright holder for this preprint (which was not certified by peer review) is the author/funder, who has granted medRxiv a license to display the preprint in perpetuity.

All rights reserved. No reuse allowed without permission.

419

420

421

422

423

424

425

426

427

428

429

430

431

432

433

434

435

436

437

438

439

440

F531L, New England BioLabs, Ipswich, MA), $0.2 \mu \mathrm{M}$ of forward and reverse primers, and about 10ng DNA templates. Negative extraction controls and blank controls with sterile water as the PCR template were included. Same volume of $1 \mathrm{X}$ loading buffer (contained SYBR green) was mixed with PCR products and then electrophoresis was operated on $2 \%$ agarose gel for detection. Samples with bright main strip between 400-450bp were chosen for further experiments. PCR products were pooled in equimolar ratios. Then, the mixture was purified with GeneJET Gel Extraction Kit (Cat No. K0691, Thermo Fisher Scientific, Waltham, MA). Sequencing libraries were constructed using NEB Next Ultra DNA Library Prep Kit for Illumina (Cat No. E7370L, New England BioLabs, Ipswich, MA) following manufacturer's recommendations. The library quality was assessed on the Agilent Bioanalyzer 2100 system (Agilent Technologies, Santa Clara, CA). At last, the library was sequenced on an Illumina HiSeq2500 platform (Illumina, San Diego, CA) and 250bp paired-end reads were generated.

\section{Bioinformatics and statistical analysis}

Paired-end reads were merged into long sequences based on the overlaps between reads 1 and reads2 by using FLASH [29]. Merged sequences then were analyzed using

QIIME 1.9.1 software package [30]. First, sequences were filtered by QIIME quality filters. Then we used pick_de_novo_otus.py to pick OTUs in addition to generate an OTU table. Sequences with a $\geq 97 \%$ similarity were assigned to the same OTUs. A representative sequence was picked for each OTU and the RDP database was used to generate taxonomic information for each representative sequence. 
441 To compute Alpha diversity, the OTU table was rarified and five metrics 442 including Chao1, observed species, PD whole tree (Faith's Phylogenetic Diversity, 443 which adds up all the branch lengths of the phylogenetic tree as a measure of 444 diversity), Shannon and Simpson were calculated. Rarefaction curves were generated 445 based on these metrics. Both weighted and unweighted unifrac distances were 446 calculated for principal coordinate analysis (PCoA). The pairwise dissimilarity 447 between the microbial community structures was assessed using Bray-Curtis distance 448 at the OTU level as described before [31]. The difference in microbial markers was 449 measured using Mann-Whitney rank test and LEfSe. When multiple hypothesis tests 450 were performed simultaneously, $P$ values were corrected using Benjamini and 451 Hochberg's false discovery rate (FDR). For the comparative analysis, only the genera and species with the abundance of $>1$ and $>0.2 \%$, respectively, in at least one of the samples were included.

The clustering of CSTs was done using complete linkage hierarchical clustering with five clusters as described by Ravel et al $[18,20]$. CST I, CST II, CST III and

456 CST V was predominated with L. crispatus, L. gasseri, L. iners, and L. jensenii, 457 respectively. CST IV was defined as lacking Lactobacillus spp. and comprising a 458 diverse set of strict and facultative anaerobes, and was further split into CST IV-A 459 and CST IV-B.

\section{Ethical statements}

461 The study was performed with the approval of the Ethical Committee of Beijing 462 Institute of Microbiology and Epidemiology and conducted according to the 
medRxiv preprint doi: https://doi.org/10.1101/2020.07.07.20148536; this version posted July 10, 2020. The copyright holder for this preprint

(which was not certified by peer review) is the author/funder, who has granted medRxiv a license to display the preprint in perpetuity.

All rights reserved. No reuse allowed without permission.

463 principles expressed in the Declaration of Helsinki. All participants provided

464 informed consent.

465 Data availability

466 The sequencing data has been submitted to GSA at the National Genomics Data

467 Center, with the accession ID CRA002692.

468 Authors' contributions

469 FZ and WL conceived the study. XZ and JW designed the study and interpreted the 470 data. XZ and JW wrote the paper. QZ and ZG collected the samples and conducted

471 the experiments. XZ, JW, XM, BX, HF, FZ and WL analyzed the data and created the

472 graphs. All authors approved the final version of the manuscript.

473 Competing interest

474 The authors have declared no competing interests.

475 Acknowledgments

476 This work was supported by the National Natural Science Foundation of China

477 (81825019, 31722031, 31670119, 31870107), the Beijing Leading Talents in Science

478 and Technology (Z181100006318008), the China Mega-Project on Infectious Disease

479 Prevention (2018ZX10713002-002, 2018ZX10101003-002, 2018ZX10301401), and

480 the National Key Research and Development Program of China (2016YFC1000705).

481 We are grateful to all the subjects, their families, and collaborating clinicians for their

482 participation.

\section{References}


[1] Ding T, Schloss PD. Dynamics and associations of microbial community types across the human body. Nature 2014;509:357-60.

[2] Reid G. Therapeutic Opportunities in the Vaginal Microbiome. Microbiol Spectr 2017;5.

[3] Millar MR. The relationship between the vaginal microbiome and human health. BJOG 2017;124:70.

[4] Romero R, Hassan SS, Gajer P, Tarca AL, Fadrosh DW, Bieda J, et al. The vaginal microbiota of pregnant women who subsequently have spontaneous preterm labor and delivery and those with a normal delivery at term. Microbiome 2014;2:18.

[5] DiGiulio DB, Callahan BJ, McMurdie PJ, Costello EK, Lyell DJ, Robaczewska A, et al. Temporal and spatial variation of the human microbiota during pregnancy. Proc Natl Acad Sci U S A

2015;112:11060-5.

[6] Kindinger LM, Bennett PR, Lee YS, Marchesi JR, Smith A, Cacciatore S, et al. The interaction between vaginal microbiota, cervical length, and vaginal progesterone treatment for preterm birth risk. Microbiome 2017;5:6.

[7] Callahan BJ, DiGiulio DB, Goltsman DSA, Sun CL, Costello EK, Jeganathan P, et al. Replication and refinement of a vaginal microbial signature of preterm birth in two racially distinct cohorts of US women. Proc Natl Acad Sci U S A 2017;114:9966-71.

[8] Romero R, Hassan SS, Gajer P, Tarca AL, Fadrosh DW, Nikita L, et al. The composition and stability of the vaginal microbiota of normal pregnant women is different from that of non-pregnant women. Microbiome 2014;2:4.

[9] Wang J, Zheng J, Shi W, Du N, Xu X, Zhang Y, et al. Dysbiosis of maternal and neonatal microbiota associated with gestational diabetes mellitus. Gut 2018;67:1614-25.

[10] Serrano MG, Parikh HI, Brooks JP, Edwards DJ, Arodz TJ, Edupuganti L, et al. Racioethnic diversity in the dynamics of the vaginal microbiome during pregnancy. Nat Med 2019;25:1001-11. [11] Fettweis JM, Serrano MG, Brooks JP, Edwards DJ, Girerd PH, Parikh HI, et al. The vaginal microbiome and preterm birth. Nat Med 2019;25:1012-21.

[12] Lumbiganon P, Laopaiboon M, Gulmezoglu AM, Souza JP, Taneepanichskul S, Ruyan P, et al. Method of delivery and pregnancy outcomes in Asia: the WHO global survey on maternal and perinatal health 2007-08. Lancet 2010;375:490-9.

[13] Huang K, Tao F, Raven J, Liu L, Wu X, Tang S. Utilization of antenatal ultrasound scan and implications for caesarean section: a cross-sectional study in rural Eastern China. BMC Health Serv Res 2012;12:93.

[14] Hu Y, Tao H, Cheng Z. Caesarean Sections in Beijing, China - Results from a Descriptive Study. Gesundheitswesen 2016;78:e1-5.

[15] Liu L, Oza S, Hogan D, Chu Y, Perin J, Zhu J, et al. Global, regional, and national causes of under-5 mortality in 2000-15: an updated systematic analysis with implications for the Sustainable Development Goals. Lancet 2016;388:3027-35. [16] Blencowe H, Cousens S, Oestergaard MZ, Chou D, Moller AB, Narwal R, et al. National, regional, and worldwide estimates of preterm birth rates in the year 2010 with time trends since 1990 for selected countries: a systematic analysis and implications. Lancet 2012;379:2162-72. [17] Zhao X, Chen Y, Qiu G, Xiao M, Zhong N. Reducing preterm births in China. Lancet 2012;380:1144-5; author reply 5.

[18] Ravel J, Gajer P, Abdo Z, Schneider GM, Koenig SS, McCulle SL, et al. Vaginal microbiome of reproductive-age women. Proc Natl Acad Sci U S A 2011;108 Suppl 1:4680-7. 
[19] Gajer P, Brotman RM, Bai GY, Sakamoto J, Schutte UME, Zhong X, et al. Temporal dynamics of the human vaginal microbiota. Science Translational Medicine 2012;4:132ra52. [20] MacIntyre DA, Chandiramani M, Lee YS, Kindinger L, Smith A, Angelopoulos N, et al. The vaginal microbiome during pregnancy and the postpartum period in a European population. Sci Rep 2015;5:8988.

[21] Dominguez-Bello MG, Costello EK, Contreras M, Magris M, Hidalgo G, Fierer N, et al. Delivery mode shapes the acquisition and structure of the initial microbiota across multiple body habitats in newborns. Proc Natl Acad Sci U S A 2010;107:11971-5.

[22] Zhang M, Ma W, Zhang J, He Y, Wang J. Analysis of gut microbiota profiles and microbe-disease associations in children with autism spectrum disorders in China. Sci Rep 2018;8:13981.

[23] Schink M, Konturek PC, Tietz E, Dieterich W, Pinzer TC, Wirtz S, et al. Microbial patterns in patients with histamine intolerance. J Physiol Pharmacol 2018;69.

[24] Zhang J, Zhang F, Zhao C, Xu Q, Liang C, Yang Y, et al. Dysbiosis of the gut microbiome is associated with thyroid cancer and thyroid nodules and correlated with clinical index of thyroid function. Endocrine 2018.

[25] Chen X, Winckler B, Lu M, Cheng H, Yuan Z, Yang Y, et al. Oral Microbiota and Risk for Esophageal Squamous Cell Carcinoma in a High-Risk Area of China. PLoS One 2015;10:e0143603. [26] Ma ZS, Li L. Quantifying the human vaginal community state types (CSTs) with the species specificity index. PeerJ 2017;5:e3366.

[27] Huang YE, Wang Y, He Y, Ji Y, Wang LP, Sheng HF, et al. Homogeneity of the vaginal microbiome at the cervix, posterior fornix, and vaginal canal in pregnant Chinese women. Microb Ecol 2015;69:407-14.

[28] Witkin SS. Vaginal microbiome studies in pregnancy must also analyse host factors. BJOG 2019;126:359.

[29] Magoc T, Salzberg SL. FLASH: fast length adjustment of short reads to improve genome assemblies. Bioinformatics 2011;27:2957-63.

[30] Caporaso JG, Kuczynski J, Stombaugh J, Bittinger K, Bushman FD, Costello EK, et al. QIIME allows analysis of high-throughput community sequencing data. Nat Methods 2010;7:335-6.

[31] Wang J, Jia Z, Zhang B, Peng L, Zhao F. Tracing the accumulation of in vivo human oral microbiota elucidates microbial community dynamics at the gateway to the GI tract. Gut 2019. 


\section{$561 \quad$ Figure legends}

562 Figure 1. The vaginal microbiome during pregnancy and postpartum was 563 significantly different in microbial diversity, community structure and 564 composition. (A) Alpha diversity of the vaginal microbiome between pregnancy and 565 postpartum. Each box plot represents the median, interquartile range, minimum, and maximum values. (B) Weighted ANOSIMs and principal coordinate analysis (PCoA) analysis of the vaginal microbiome during pregnancy and postpartum based on the distance matrix of UniFrac dissimilarity. The $\mathrm{x}$ - and $\mathrm{y}$-axes represent two dimensions explaining the greatest proportion of variance in the communities. Each dot represents a sample, and each circle shows a 95\% confidence interval. (C) Relative abundance of the genus level. The discriminating taxa were identified based on linear discriminant analysis effect size (LEfSe) analysis with a threshold of linear discriminant analysis

575 (LDA) scores $(\log 10)>2$ and $P<0.05$. The prefixes $\mathrm{p}_{-}, \mathrm{c}_{-}, \mathrm{o}_{-}, \mathrm{f}_{-}, \mathrm{g}_{-}, \mathrm{s}_{-}$represent 576 phylum, class, order, family, genus, and species respectively. vaginal microbiome during pregnancy between pregnant women with (HBP) and without hypertensive disorders (without HBP). Each box plot represents the median, 581 interquartile range, minimum, and maximum values. (B) Cladogram using LEfSe indicates the phylogenetic distribution of vaginal microbiome associated with 
583

584

585

586

587

588

pregnant women who had hypertensive disorders with pregnancy. (C) Weighted

ANOSIMs based on the distance matrix of UniFrac dissimilarity of the vaginal microbiome during pregnancy in pregnant women with or without abortion history. The axes represent the two dimensions explaining the greatest proportion of variance in the communities. Each dot represents a sample, and each circle shows a $95 \%$ confidence interval. (D) Cladogram using LEfSe indicates the phylogenetic distribution of vaginal microbiome associated with pregnant women who had history of abortion. The LDA scores $(\log 10)>2$ and $P<0.05$ are listed. The prefixes $\mathrm{p}_{-}, \mathrm{c}_{-}$, $\mathrm{o}_{-}, \mathrm{f}_{-}, \mathrm{g}_{-}, \mathrm{s}_{-}$represent phylum, class, order, family, genus, and species respectively.

\section{Figure 3. The vaginal microbiome postpartum varied by delivery mode and age}

of the pregnant woman. (A) Alpha diversity of the vaginal microbiome during pregnancy between the pregnant women who delivered naturally or cesarean section in the postpartum period. Each box plot represents the median, interquartile range, minimum, and maximum values. (B) Weighted ANOSIMs based on the distance matrix of UniFrac dissimilarity of the vaginal microbiome in the pregnant women who delivered naturally or cesarean section in the postpartum period. The axes represent the two dimensions explaining the greatest proportion of variance in the communities. Each dot represents a sample, and each circle shows a 95\% confidence interval. (C) Cladogram using LEfSe indicates the phylogenetic distribution of vaginal microbiome associated with pregnant women who delivered naturally or cesarean section in the postpartum period. (D) Weighted ANOSIMs based on the distance matrix of UniFrac dissimilarity of the vaginal microbiome in the pregnant 
605

606

607

608

609

610

611

612

613

614

615

616

617

618

619

620

621

622

623

624

625

women who had advanced or young age in the postpartum period. (E) Cladogram using LEfSe indicates the phylogenetic distribution of vaginal microbiome associated with pregnant women who had advanced or young age in the postpartum period. The LDA scores $(\log 10)>2$ and $P<0.05$ are listed. The prefixes $\mathrm{p}_{-}, \mathrm{c}_{-}, \mathrm{o}_{-}, \mathrm{f}_{-}, \mathrm{g}_{-}, \mathrm{s}_{-}$ represent phylum, class, order, family, genus, and species respectively.

\section{Figure 4. The abundance of specific vaginal bacteria changed in multiple groups}

during pregnancy and postpartum. (A) The relative abundances of the 21 bacterial taxa was varied significantly in two comparisons simultaneously during pregnancy and in postpartum period based on LEfSe analysis. The discriminating taxa were identified with a threshold of LDA scores $(\log 10)>2$ and $P<0.05$. The boxes filled in blue and red color represent the discriminating taxa enriched and depleted in the vaginal microbiome of women who delivered cesarean, with advanced maternal age, overweight, hypertensive disorders or other pregnancy complications, and without abortion history during pregnancy and postpartum, respectively. (B) The relative abundance of Lactobacillaceae family in pregnant women who delivered cesarean section and advanced maternal age. (C) The relative abundance of Lactobacillus iners in pregnant women without abortion history during pregnancy and those with advanced maternal age in postpartum. (D) The relative abundance of Lactobacillus reuteri in women without abortion history and young maternal age in postpartum. The prefixes $\mathrm{p}_{-}, \mathrm{c}_{-}, \mathrm{o}_{-}, \mathrm{f}_{-}, \mathrm{g}_{-}, \mathrm{s}_{-}$represent phylum, class, order, family, genus, and species respectively. 

microbiome was different corresponding to different maternal factors. The percentage of each CST in each group was represented by one color. The top three bars showed the mean of CST percentage in samples of all, during pregnancy, and postpartum period, respectively. Term denotes the pregnant woman who delivered in term, Term-PROM denotes premature rupture of membranes (PROM) occurred but birth in term at last, PROM-preterm denotes PROM complicated with preterm birth (PTB), and Non-PROM-preterm denotes without PROM occurred but premature birth at last.

Table S1. Characteristics of pregnant women included in the study (DOCX 16 kb)

Table S2. The significantly different bacteria between pregnancy and postpartum (XLSX 20 kb)

Table S3. Distribution of community state types according to delivery mode, age, abortion, BMI, pregnancy and delivery complications (DOCX 25 kb) significantly lower than those of postpartum. Differences in the $\alpha$ diversities of the vaginal microbiome between pregnancy and postpartum according to the observed 
648 index. Each box plot represents the median, interquartile range, minimum, and 649 maximum values.

650 Figure S2. The vaginal microbiome of pregnant women was more similar to each 651 other during pregnancy. Weighted and unweighted PCoA (A-B) and ANOSIM (C-D) 652 based on the distance matrix of UniFrac dissimilarity of the vaginal microbial 653 communities in pregnancy and postpartum. Respective ANOSIM R values show the 654 community variation between the compared groups, and significant $P$ values are 655 indicated. The axes represent the two dimensions explaining the greatest proportion of 656 variance in the communities. Each dot represents a sample.

657 Figure S3. The vaginal microbiome of most women after delivery was classified 658 as CST IV-A. Color bar from yellow to red shows the relative abundance of each 659 microbial taxon. The prefixes $g_{-}, s_{-}$represent genus, and species respectively.

Figure S4. Little difference was shown between early and late stages of pregnancy in microbial diversity, community structure and composition of the vaginal microbiome. (A-E) Differences in the microbial diversities of the vaginal 663 microbiome between early and late stages according to the Chao 1, observed species, PD whole tree, Shannon and Simpson index. (F) Weighted ANOSIMs and PCoA based on the distance matrix of UniFrac dissimilarity of the vaginal microbiome of early and late pregnant stages. (G) Relative abundance of the discriminating taxa in 667 the vaginal microbiome between early and late pregnant stages at all levels. $(\mathrm{H})$ 668 Relative abundance of the discriminating taxa in the vaginal microbiome of early and 
670

671

672

on LEfSe analysis with a threshold of LDA scores $(\log 10)>2$ and $P<0.05$. The prefixes $p_{-}, c_{-}, o_{-}, f_{-}, g_{-}, s_{-}$represent phylum, class, order, family, genus, and species respectively.

\section{Figure S5. LEfSe analysis showed the discriminant bacteria of vaginal} microbiome during pregnancy corresponding to multiple complex factors. (A) Different delivery mode. (B) Advanced maternal age vs. young maternal age. (C) With vs. without gestational diabetes mellitus. (D) With vs. without hypothyroidism. The LDA scores $(\log 10)>2$ and $P<0.05$ are listed. The prefixes $\mathrm{p}_{-}, \mathrm{c}_{-}, \mathrm{o}_{-}, \mathrm{f}_{-}, \mathrm{g}_{-}, \mathrm{s}_{-}$ represent phylum, class, order, family, genus, and species respectively.

Figure S6. LEfSe analysis showed the discriminant bacteria of vaginal microbiome in postpartum corresponding to multiple complex factors. (A) Different body mass index before pregnancy. (B) With vs. without abortion history. (C) With vs. without pregnancy complications. The LDA scores $(\log 10)>2$ and $P<$ 0.05 are listed. The prefixes $c_{-}, o_{-}, f_{-}, g_{-}, s_{-}$represent class, order, family, genus, and species respectively.

Figure S7. Most discriminant bacteria of the vaginal microbiome were different under complex factors during pregnancy and postpartum. The relative abundance of 163 bacterial taxa, including 5 phyla, 12 classes, 22 orders, 35 families, 86 genera, and 3 species, varied significantly between groups during pregnancy and postpartum based on LEfSe analysis. The LDA scores $(\log 10)>2$ and $P<0.05$ are listed. The boxes filled in blue and red color represent the discriminating taxa enriched and depleted in the vaginal microbiome of women who delivered cesarean, with advanced 
692 maternal age, overweight, hypertensive disorders, hypothyroidism or other pregnancy

693

694

695

696

697

698

699

700

701

702

703

704 complications, and without abortion history or gestational diabetes mellitus during

pregnancy and postpartum, respectively. The prefixes $\mathrm{p}_{-}, \mathrm{c}_{-}, \mathrm{o}_{-}, \mathrm{f}_{-}, \mathrm{g}_{-}, \mathrm{s}_{-}$represent

phylum, class, order, family, genus, and species respectively.

Figure S8. PTB and PROM were accompanied by some bacteria enrichment in

the vaginal microbiome. Based on LEfSe analysis, the relative abundances of 68

bacterial taxa varied significantly between groups. The LDA scores $(\log 10)>2$ and $P$

$<0.05$ are listed. (A) Preterm vs. term group during pregnancy. (B) PROM vs. term group during pregnancy. (C) PROM related preterm vs. term group during pregnancy.

(D) Non-PROM related preterm vs. term group during pregnancy. (E) PROM related preterm vs. non-PROM related preterm during pregnancy. (F) Preterm vs. term group in postpartum. (G) PROM vs. term group in postpartum. The prefixes $p_{-}, c_{-}, o_{-}, f_{-}$, g_, s_ represent phylum, class, order, family, genus, and species respectively. 
A

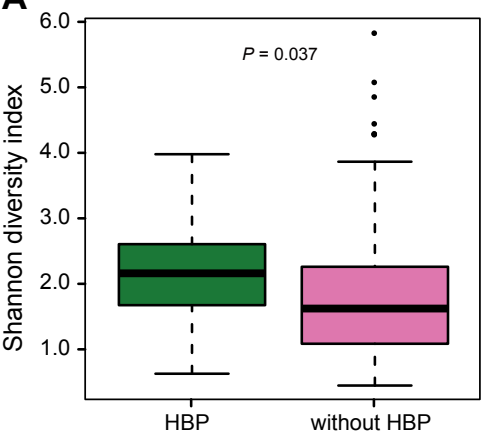

B

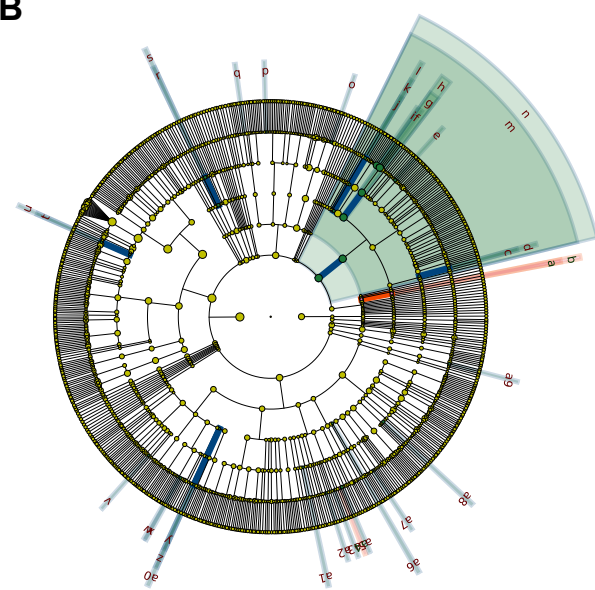

C

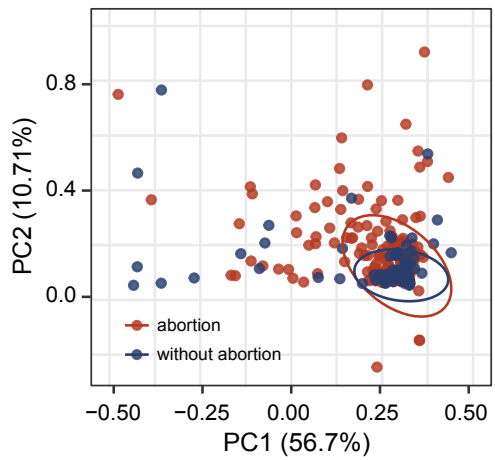

D

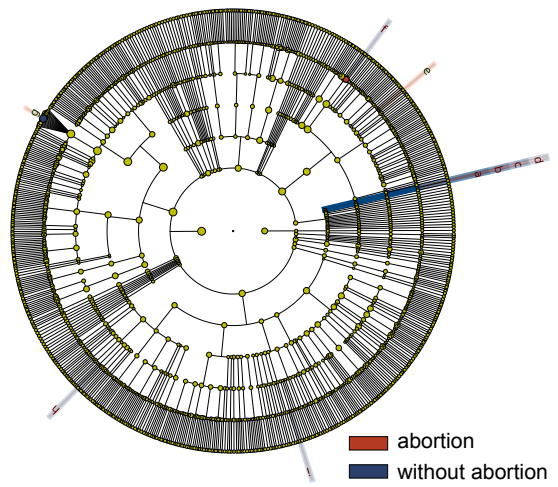

HBP

$\square$ without HBP

$\square$ a: g_Gp5
b: c_Acidobacteria_Gp5
c: g__lamia
d: f_lamiaceae
e: g_Saccharomonospora
f: g_Gardnerella
g: f_Bifidobacteriaceae
h: o_Bifidobacteriales
i: g_Atopobium
j: g_Euzebya
k: f_Euzebyaceae
l: o_Euzebyales
m: c_Actinobacteria
n: p_Actinobacteria
o: g_Butyricimonas
p: g_Vitellibacter
q: g_Haliscomenobacter
r: g_GpXI

$\square$ a: g_Geothrix

b: f_Holophagaceae

$\square$ c: o_Holophagales

$\square \mathrm{d}: \mathrm{c}$ _Holophagae

e: g_Kribbella

$\square$ f: g_Bifidobacterium

g: $s$ Lactobacillus iners

h: g_Terasakiella

$\square$ i: g_Cystobacter

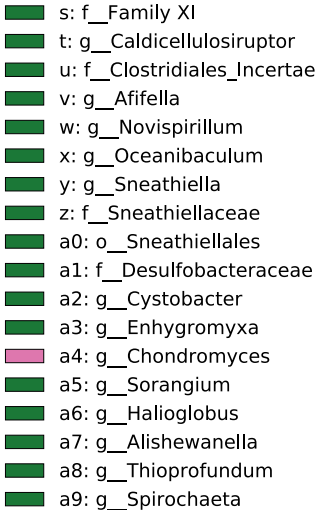

$\square$ s: f_Family XI
t: g_Caldicellulosiruptor

v: g_Afifella

x: g Oceanibaculum

y: g Sneathiella

z: f Sneathiellaceae

a1: $f$ Desulfobacteraceae

a2: g Cystobacter

a4: g Chondromyces

a5: g Sorangium

a9: g_Spirochaeta 
A

Pregnancy

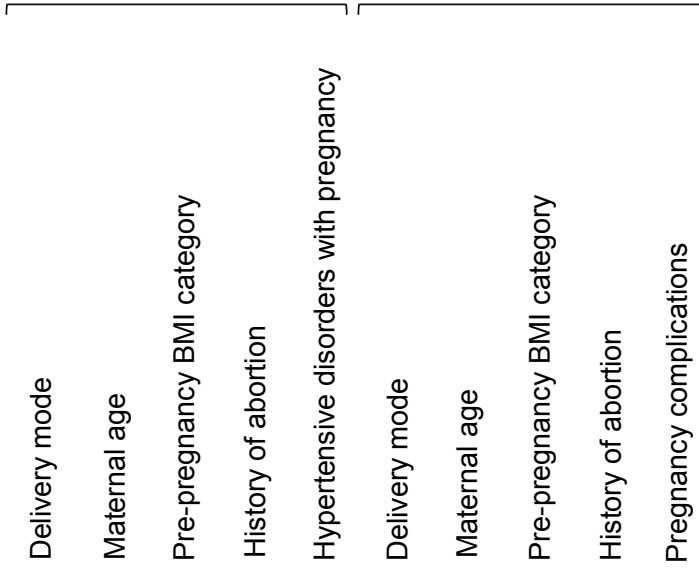

B

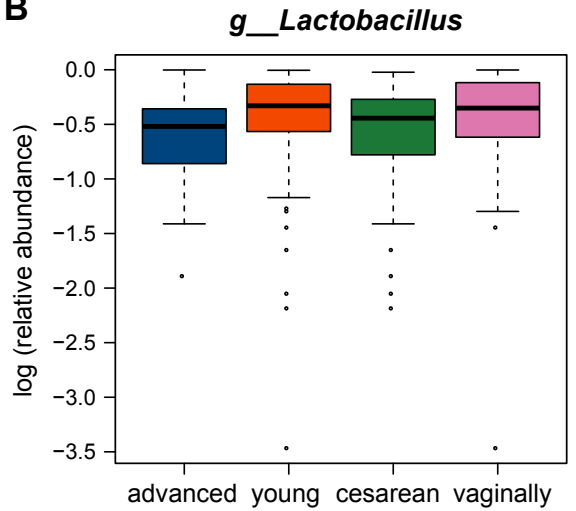

C

g__Propionibacterium

o Bifidobacteriales

f_Bifidobacteriaceae

p__ Gemmatimonadetes

c_Gemmatimonadetes

o__Gemmatimonadales

f__ Gemmatimonadaceae

g_Gemmatimonas

g_Rheinheimera

f__Chromatiaceae

o_Chromatiales

g_Klebsiella

s_Lactobacillus iners

g_Butyricimonas

$f \_$Lactobacillaceae

g_Lactobacillus

g_Sneathia

s_Lactobacillus reuteri

g_Bulleidia

g_Cellulosilyticum

g_Nosocomiicoccus
s_Lactobacillus iners

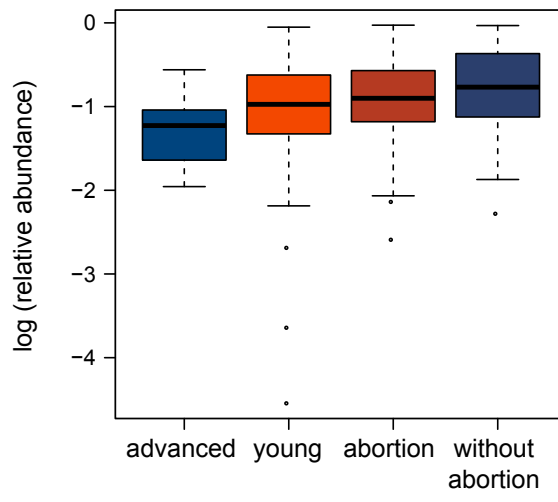

D s_Lactobacillus reuteri

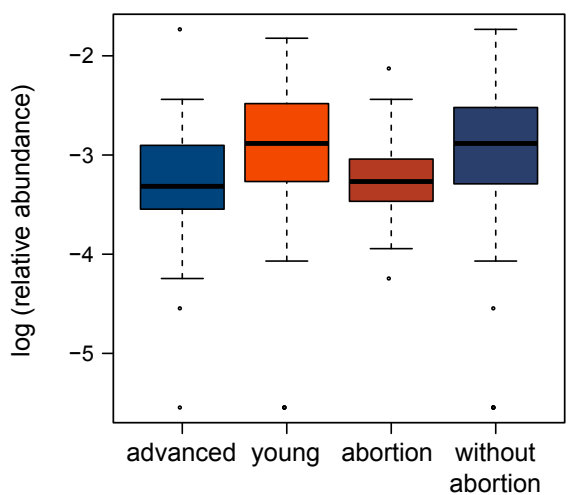


During pregnancy

Postpartum period

Cesarean section Vaginally delivered

Young maternal age Advanced maternal age

With history of abortion Without history of abortion

Normal Underweight Overweight

With pregnancy complications Without pregnancy complications

With hypertensive disorders Without hypertensive disorders

With gestational diabetes mellitus Without gestational diabetes mellitus

With hypothyroidism Without hypothyroidism

\section{Term \\ PROM \\ Term-PROM \\ Preterm \\ PROM-preterm}

Non-PROM-preterm

Cesarean section

Vaginally delivered

Young maternal age Advanced maternal age

With history of abortion Without history of abortion

Underweight

Normal

Overweight

With pregnancy complications Without pregnancy complications

With hypertensive disorders Without hypertensive disorders

With gestational diabetes mellitus Without gestational diabetes mellitus

With hypothyroidism Without hypothyroidism

Term

PROM

Term-PROM

Preterm

PROM-preterm

Non-PROM-preterm 